\title{
Editorial
}

\section{Modeling and Control Problems in Sustainable Transportation and Power Systems}

\author{
Xiaosong Hu, ${ }^{1,2,3}$ Shengbo Eben $\mathrm{Li}^{4}{ }^{4}$ and Dongsuk Kum ${ }^{5}$ \\ ${ }^{1}$ The State Key Laboratory of Mechanical Transmissions, Chongqing University, Chongqing 400044, China \\ ${ }^{2}$ College of Automotive Engineering, Chongqing University, Chongqing 400044, China \\ ${ }^{3}$ Department of Civil and Environmental Engineering, University of California, Berkeley, CA 94720, USA \\ ${ }^{4}$ The State Key Laboratory of Automotive Safety and Energy, Tsinghua University, Beijing 100084, China \\ ${ }^{5}$ The Cho Chun Shik Graduate School for Green Transportation, Korea Advanced Institute of Science and Technology (KAIST), \\ Daejeon 305-701, Republic of Korea \\ Correspondence should be addressed to Xiaosong Hu; xiaosonghu@ieee.org
}

Received 18 April 2016; Accepted 19 April 2016

Copyright (C) 2016 Xiaosong Hu et al. This is an open access article distributed under the Creative Commons Attribution License, which permits unrestricted use, distribution, and reproduction in any medium, provided the original work is properly cited.

Sustainable transportation and power systems have the great potential to significantly reduce hydrocarbon consumptions, pollutant emissions, and carbon footprint [1]. The two key energy sectors are intimately coupled. For instance, largescale deployment of electrified vehicles is considerably beneficial to renewable ways of power generation by including wind and solar energy sources. Such good synergies between the transportation and power sectors expedite a revolutionary transition towards a clean, high-efficiency, and affordable energy future. The inevitably increasing system complexity and the desire to make the most of sustainable energy systems constitute a major incentive to leverage advanced approaches of system modeling, simulation, control, optimization, diagnosis, and prognostics. In systemlevel analysis, there are various modeling/control challenges surrounding transportation electrification (e.g., alternativeenergy powertrains), intelligent transportation system (e.g., autonomous driving and connected vehicles), smart grid (e.g., microgrids and renewables integrations), and vehicletraffic-grid-building interactions. A wealth of optimization methods has been proposed to devise sophisticated energy management strategies for tackling such challenges, including dynamic programming (DP) [2], equivalent consumption minimization strategy (ECMS) [3], Pontryagin's minimum principle (PMP) [4], convex programming [5], and model predictive control [6]. In component-level analysis, there are also plentiful modeling/control challenges surrounding energy storage systems (e.g., batteries, ultracapacitors, fuel cells, flywheels, and hybrid storage schemes), electric machine/motor drive systems, V2I/V2V communication, and power electronic circuits, and so forth. For example, diverse approaches have been utilized to establish highfidelity battery/ultracapacitor models, including offline particle swarm optimization (PSO) algorithm, genetic algorithm, fractional-order calculus, and online extended Kalman filtering. A plethora of battery/ultracapacitor state estimation and charging control techniques has also been reported in the recent literature [7-9].

The main goal of this special issue is to provide a professional platform sharing timely, advanced solutions to modeling, simulation, control, optimization, and fault diagnosis/prognosis of sustainable transportation and power systems. Particular emphasis is placed on systemlevel/component-level modeling, optimization, control, and fault diagnosis/prognosis.

For this special issue, 84 submissions have been totally received. After rigorous review processes, 37 manuscripts have been ultimately accepted, which cover a broad range of key modeling/control problems in sustainable transportation and power systems. A brief snapshot of them is given below.

W. Song et al. proposed an intention-aware autonomous driving decision making algorithm for an uncontrolled 
intersection scenario. Y. Ji et al. developed a feedback gating control policy for mitigating network congestion by adjusting signal timings of gating intersections, based on a macroscopic fundamental diagram. J. Heng et al. devised a hybrid power load forecasting method, which comprises empirical mode decomposition, Cuckoo search algorithm, and wavelet neural network. P. D. U. Coronado and H. Ahuett-Garza synthesized a rule-based control strategy for a dual motor propulsion system with a differential transmission. K. Hwang et al. reported an autonomous coil alignment system using fuzzy steering control for electric vehicles with dynamic wireless charging. Z. Chen et al. discussed an online energy management strategy of plug-in hybrid electric vehicles for prolongation of all electric range based on dynamic programming. $\mathrm{H}$. Li et al. proposed an optimization scheduling model for wind power and thermal power with energy storage system considering carbon emission trading. S. Zhu et al. introduced a genetic algorithm-based micro-scale vehicle emission model. X. Gong et al. presented a bus travel time deviation analysis using automatic vehicle location data and structural equation modeling. J. Zhao et al. delivered a dynamic model of Kaplan turbine regulating system which is suitable for power system assessment. X. Wu et al. proposed a multistage CC-CV charging protocol for Li-ion batteries. C. Peng et al. presented a wind power and load forecasting method based on frequency control approach for a winddiesel-battery hybrid power system. S. Salas-Duarte et al. evaluated a trapezoidal predictive controller for a four-wire active power filter for utility equipment of metro railway power-land substations. L. Pan and C. Zhang designed a high power density integrated charger for electric vehicles with active ripple compensation. C. T. Calafate et al. handled traffic flow classification issue in traffic management as a service. D. Wang et al. used polarity comparison principle to implement ultra-high-speed travelling wave protection of transmission line, based on empirical mode decomposition. C. Lu et al. investigated intelligent ramp control for incident response by means of Dyna-Q architecture. Y.-N. Zhao et al. proposed an entropy-cost function evaluation method for unmanned ground vehicles. H. Wang et al. presented dynamic modeling and control strategy optimization of a hybrid electric tracked vehicle. T. Zhao et al. devised a robust online SOC estimator for a lithium-ion battery pack based on error sensitivity analysis. J. Liu et al. built a modified quasi-steady state model of DC system in transient stability simulation under asymmetric faults. K. Yu et al. assessed model predictive energy management for connected hybrid electric vehicles. T. Chen et al. conducted a loss prediction and thermal analysis of surface-mounted brushless AC PM machines for electric vehicle application considering driving duty cycle. Y. Li et al. presented a geometric-process-based battery management optimization policy for an electric bus. H. Tao et al. constructed a small-signal model of marine electromagnetic detection transmitter controlled-source circuit. J. Wang et al. studied the effect of the integrated service mode and travel time uncertainty on taxis network equilibrium. J. Gao and $\mathrm{H}$. He compared several nonlinear filtering methods for SOC estimation of $\mathrm{a} \mathrm{Li}_{4} \mathrm{Ti}_{5} \mathrm{O}_{12} \mathrm{Li}$-ion battery. P. Jiang and Q. Dong proposed a new hybrid model for wind speed prediction using an intelligent optimization algorithm and a data denoising method. F. Chang et al. elucidated fault characteristics and control strategies of multiterminal high voltage direct current transmission based on modular multilevel converter. Z. Shi et al. presented an improved macro model of traffic flow with the consideration of ramps and numerical tests. S. Toosi et al. studied operational modes and control strategies of a multidirectional MC for battery-based systems. X. Meng et al. presented a hybrid MMC topology with DC fault ridethrough capability for MTDC transmission system. W. Yang et al. proposed a multiperiod vehicle lease planning policy for urban freight consolidation network. X. Li et al. evaluated robustness of some SOC estimation algorithms for EV Li-ion batteries against modeling errors and measurement noise. C. Piao et al. proposed a Li-ion battery cell-balancing algorithm for battery management system based on real-time outlier detection. C. Chu et al. presented a temporal-spatial analysis of traffic congestion based on modified CTM. C. Zhang et al. examined an improved adaptive control of static synchronous compensator in power systems.

\section{Acknowledgments}

We sincerely appreciate all the authors' contributions to this special issue and reviewers' great efforts to rigorously examine manuscripts received.

Xiaosong $\mathrm{Hu}$ Shengbo Eben $\mathrm{Li}$

Dongsuk Kum

\section{References}

[1] X. Hu, C. M. Martinez, and Y. Yang, "Charging, power management, and battery degradation mitigation in plug-in hybrid electric vehicles: a unified cost-optimal approach," Mechanical Systems and Signal Processing, 2016.

[2] L. Li, C. Yang, Y. Zhang, L. Zhang, and J. Song, "Correctional DP-based energy management strategy of plug-in hybrid electric bus for city-bus route," IEEE Transactions on Vehicular Technology, vol. 64, no. 7, pp. 2792-2803, 2015.

[3] C. Musardo, G. Rizzoni, Y. Guezennec, and B. Staccia, "AECMS: an adaptive algorithm for hybrid electric vehicle energy management," European Journal of Control, vol. 11, no. 4-5, pp. 509-524, 2005.

[4] N. Kim, S. Cha, and H. Peng, "Optimal control of hybrid electric vehicles based on Pontryagin's minimum principle," IEEE Transactions on Control Systems Technology, vol. 19, no. 5, pp. 1279-1287, 2011.

[5] X. Hu, J. Jiang, B. Egardt, and D. Cao, "Advanced power-source integration in hybrid electric vehicles: multicriteria optimization approach," IEEE Transactions on Industrial Electronics, vol. 62, no. 12, pp. 7847-7858, 2015.

[6] H. Borhan, A. Vahidi, A. M. Phillips, M. L. Kuang, I. V. Kolmanovsky, and S. Di Cairano, "MPC-based energy management of a power-split hybrid electric vehicle," IEEE Transactions on Control Systems Technology, vol. 20, no. 3, pp. 593-603, 2012.

[7] X. Hu, R. Xiong, and B. Egardt, "Model-based dynamic power assessment of lithium-ion batteries considering different operating conditions," IEEE Transactions on Industrial Informatics, vol. 10, no. 3, pp. 1948-1959, 2014. 
[8] W. Waag, C. Fleischer, and D. U. Sauer, "Critical review of the methods for monitoring of lithium-ion batteries in electric and hybrid vehicles," Journal of Power Sources, vol. 258, pp. 321-339, 2014.

[9] P. Keil and A. Jossen, "Charging protocols for lithium-ion batteries and their impact on cycle life-an experimental study with different 18650 high-power cells," Journal of Energy Storage, vol. 6, pp. 125-141, 2016. 


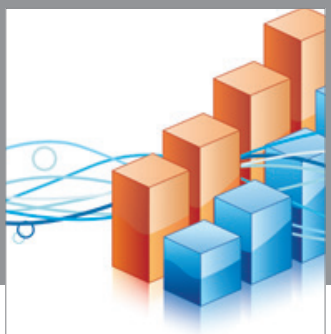

Advances in

Operations Research

vatem alat4

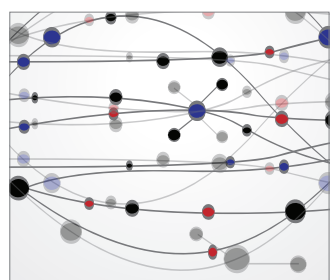

\section{The Scientific} World Journal
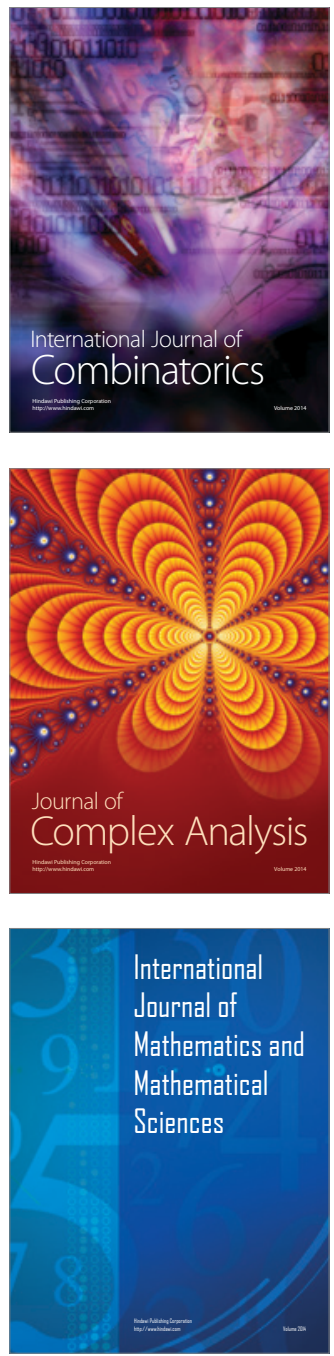
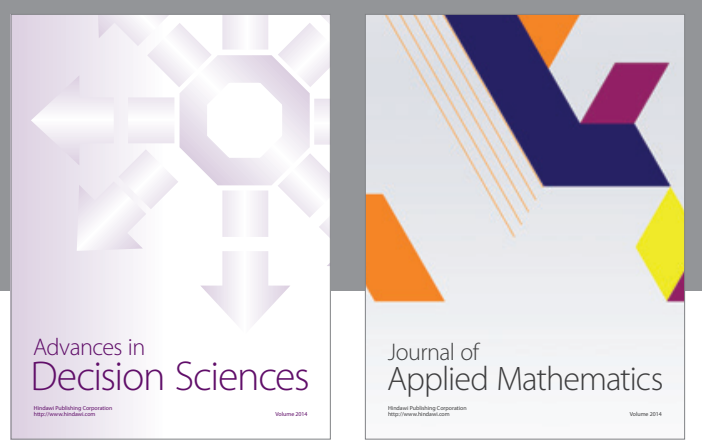

Algebra

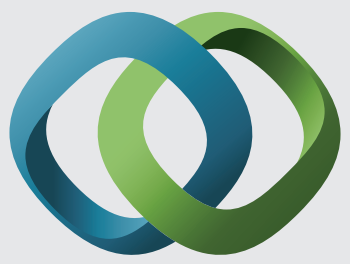

\section{Hindawi}

Submit your manuscripts at

http://www.hindawi.com
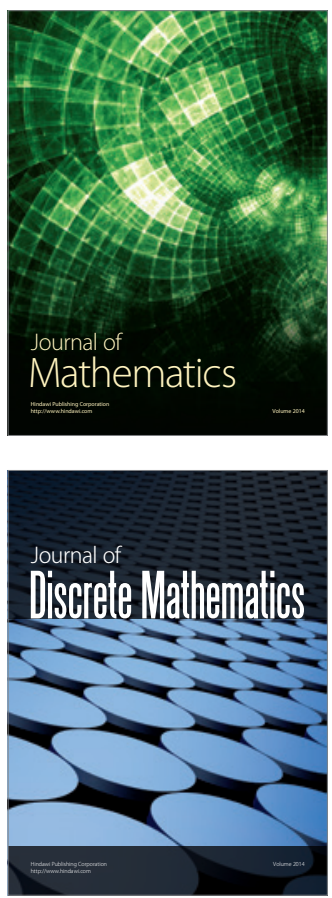

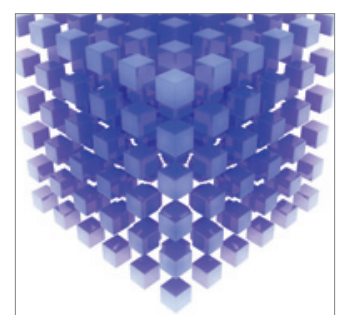

Mathematical Problems in Engineering
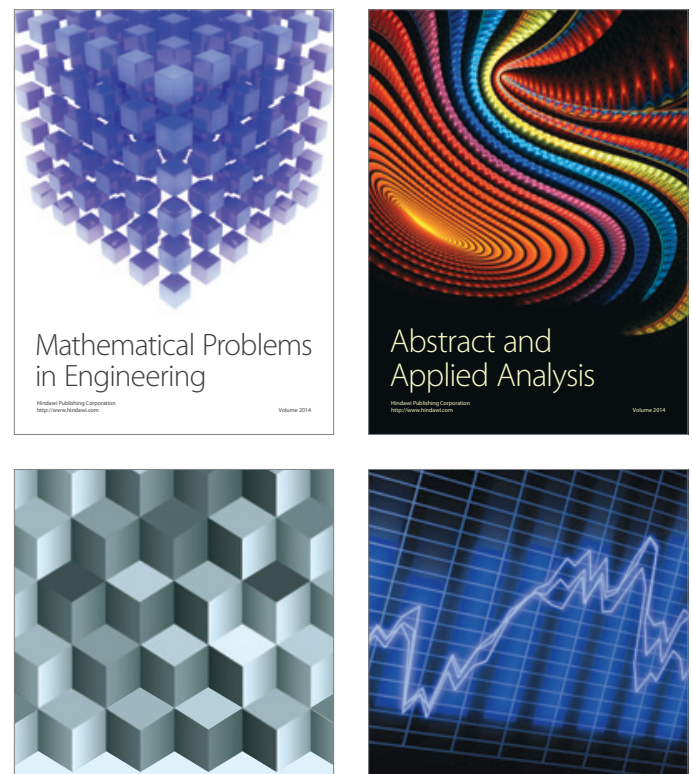

Journal of

Function Spaces

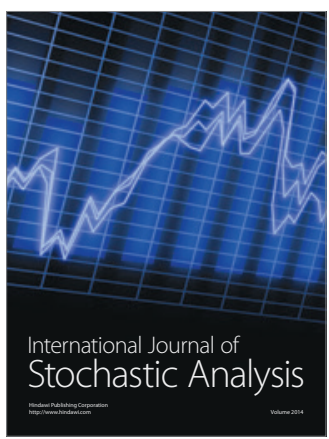

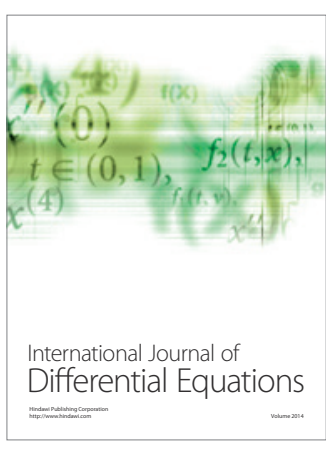
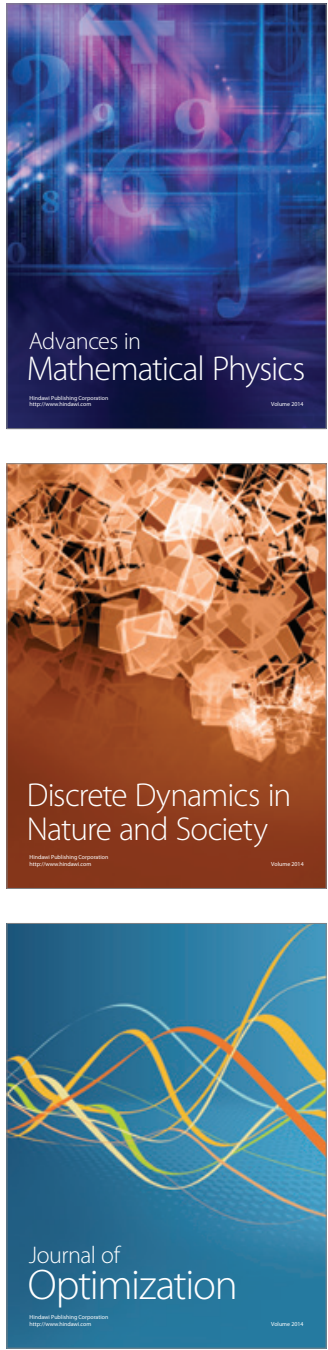